\title{
The effects of the mindfulness added to body-mind-spirit group therapy \\ (Mindfulness with BMS) on improving depressive symptoms among non-small cell lung cancer (NSCLC) patients
}

\author{
Fei-Hsiu Hsiao, RN, PhD, Professor, School of Nursing, College of Medicine, \\ National Taiwan University, Vice-Director, Department of Nursing, National \\ Taiwan University Hospital, E-mail: hsiaofei@ntu.edu.tw
}

Aims and objectives. This study aims to examine the effects of Mindfulness with BMS group therapy on improving BDI-II depressive symptoms, physical distress (EORTC QLQ-C30)(physical symptoms and functions), psychological well-being (mindfulness, Five Facet Mindfulness Questionnaire, FFMQ), holistic well-being (HWS), meaning in life (MLQ) among NSCLC patients.

Methods. This study adopts the randomized controlled trial (RCT) design. Total 62 patients who are the stage 0-IIIA of non-small cell lung cancer (NSCLC). The subjects in a control group receive 120 minutes every week for 2-month BMS group. Subjects in experimental group receive 120 minutes every week for 2-month mindfulness with BMS group. Mindfulness aim to disengage rumination and attention at the present moment reality. Practices include attitudes of mindfulness (Non-judging, Acceptance, Letting go), body scan, breathing with imaging self-compassion, compassion from others, and compassion for others.
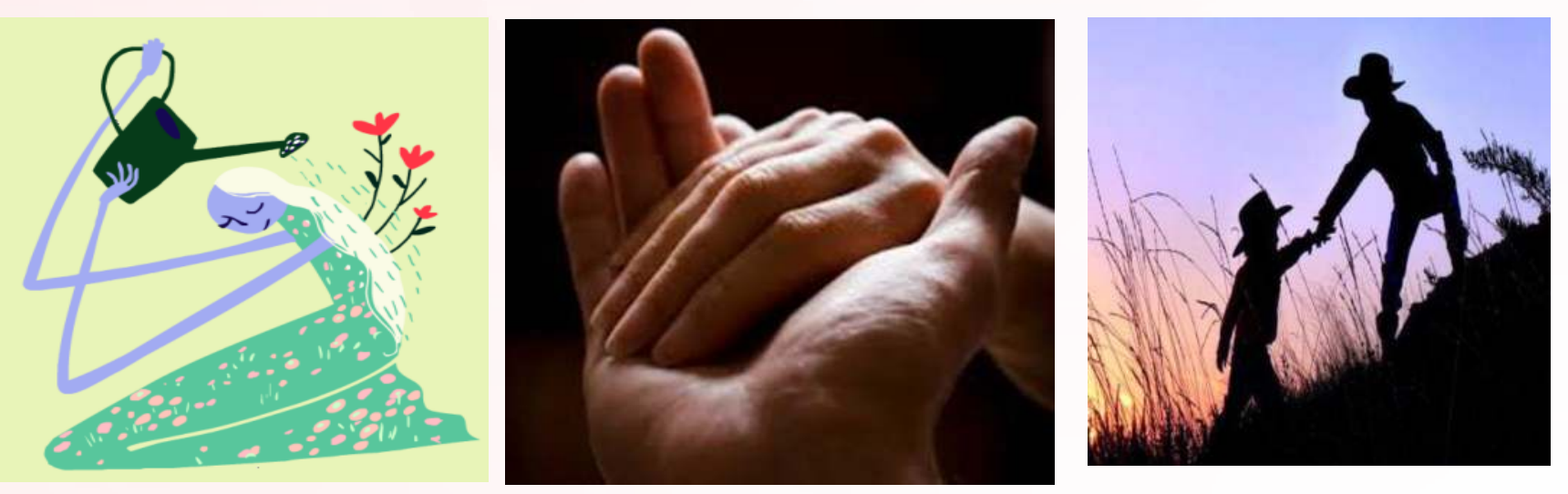

\section{Results \& Conclusions.}

The results suggest BMS could decrease depression levels for the participants in both experimental and control groups during the 5-month follow ups. Additional mindfulness to BMS group therapy could reduce emotional vulnerability and spiritual disorientation, and increase global health status and mindfulness levels. Mindfulness with BMS group therapy can be applied to increase holistic well-being for NSCLC patients
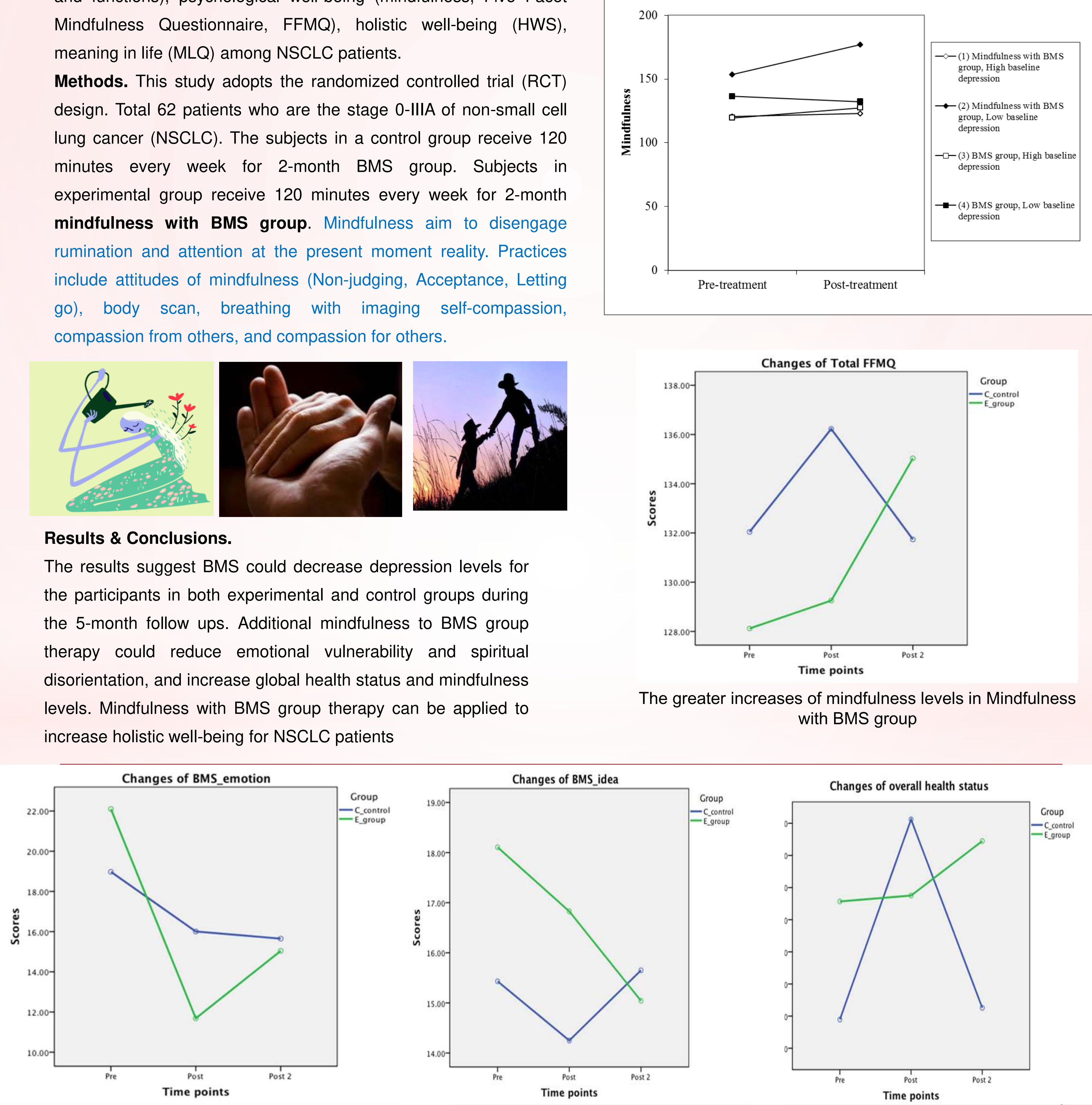

The greater reductions in emotions vulnerability and spiritual disorientation, the greater increases of global health scores in Mindfulness with BMS group 